\title{
Exploring the concepts and practices of advanced breast cancer treatment: a narrative review
}

\author{
Xiaojia Wang $^{1,2}$, Xiying Shao ${ }^{1,2}$, Jian Huang $^{1,2}$, Lei Lei $^{1,2}$, Yuan Huang ${ }^{1,2}$, Yabing Zheng ${ }^{1,2}$, Wenming Cao ${ }^{1,2}$, \\ Zhanhong Chen ${ }^{1,2}$
}

${ }^{1}$ Department of Medical Oncology (Breast Cancer), Cancer Hospital of the University of Chinese Academy of Sciences/Zhejiang Cancer Hospital, Hangzhou, China; ${ }^{2}$ Institute of Cancer and Basic Medicine (ICBM), Chinese Academy of Sciences, Hangzhou, China

Contributions: (I) Conception and design: X Wang; (II) Administrative support: X Wang, X Shao; (III) Provision of study materials or patients: J Huang, Z Chen; (IV) Collection and assembly of data: Y Zheng, W Cao; (V) Data analysis and interpretation: L Lei, Y Huang; (VI) Manuscript writing: All authors; (VII) Final approval of manuscript: All authors.

Correspondence to: Zhanhong Chen. No. 1, East Banshan Road, Gongshu District, Hangzhou 310022, China Email: czred@sina.com.

Objective: To explore the concepts and practices of advanced breast cancer treatment.

Background: Metastatic breast cancer (MBC) has become a chronic disease, with a median overall survival (OS) of around 3 years and a 5 -year survival rate of about $25 \%$. OS are strongly associated with the best available care, which consists of not only application of guidelines, but also multidisciplinary specialized care, the most efficacious medicines, and so on. Advanced breast cancer (ABC) Guidelines are the most important and authoritative guidelines for MBC.

Methods: In this review, we demonstrate the history and evolution of the global ABC Guidelines. Since 2015, Chinese multidisciplinary experts have drafted guidelines for clinical diagnosis and treatment of MBC. All of these ABC guidelines describe specialized therapeutic principles for different subtypes MBC in detail. Encouragingly, we have found that some special subtypes are hopeful of being cured, such as HER2 positive patients with low tumor burden or HR-positive (HR+) MBC with non-visceral metastasis. In our opinion, the definition of cure of $\mathrm{MBC}$ is that $\mathrm{MBC}$ patients achieve $\mathrm{CR}$ and remain for more than five years after systemic treatment, including those with local therapy. Consequently, we also have conducted some researches and meaningful explorations in different subtypes of MBC. In HER2 positive MBC, our study revealed that regular HER2 circulating extracellular domain (ECD) assay can provide the real-time monitoring of tumor burden and prediction of poor outcome, and may present an important opportunity to reassess HER2 status. In HR+MBC, we suggested that hormone therapy (HT) maintenance is the priority choice for HR+/HER2 - MBC after first-line combined chemotherapy. Besides, our real-world study revealed that fulvestrant combined with ovarian suppression was an active option for premenopausal HR+/HER2- MBC. And also, we observed that everolimus (low-dose) combined with hormone therapy was still effective for HR+/HER2- MBC. For mTNBC patients, we found that THA and endostatin exhibited potential efficacy and was well tolerated in pretreated patients.

Conclusions: Our concepts and practices will contribute to the design of relevant clinical research and accumulation of evidence, and cure of $\mathrm{MBC}$ is promising.

Keywords: Metastatic breast cancer (MBC); treatment; cure; molecular types; targeted therapy

Submitted Feb 03, 2021. Accepted for publication Apr 22, 2021.

doi: $10.21037 /$ atm-21-1458

View this article at: http://dx.doi.org/10.21037/atm-21-1458

^ ORCID: 0000-0002-7005-0842. 
Metastatic breast cancer (MBC) is the later stage in the development of breast cancer and often indicates that the overall survival of patients is short, with palliative treatment being the main strategy. Inoperable locally advanced breast cancer (LABC), accounts for about 5-10\% of the initial metastatic breast cancer patients, and most patients with advanced breast cancer $(\mathrm{ABC})$ are those with recurrence and metastasis from early breast cancer, who have undergone surgery and various adjuvant therapies (1). The MBC patients are often resistant to various antitumor therapies and have a myriad of complications and visceral involvement. Therefore, these patients have a poor prognosis and little hope of cure. However, in recent years, the efficacy and survival of $\mathrm{ABC}$ have been constantly improved upon due to the continuous updating of the concept of comprehensive treatment, particularly classification treatment, individualized strategies based on molecular typing, and new breakthrough drugs [e.g., fulvestrant, nab-paclitaxel, CDK4/6 inhibitors, monoclonal antibodies against human epidermal growth factor receptor 2 (HER2) drugs, tyrosine kinase inhibitors (TKIs), antibody-drug conjugates (ADCs), immune checkpoint inhibitors (ICIs)]. Breast cancer is different from other malignant tumors: in addition to the usual procedures of surgery, adjuvant chemotherapy, and radiation, other options, including, neoadjuvant chemotherapy, targeted therapy, adjuvant endocrine therapy, and targeted therapy, are available. These advances have improved the survival of breast cancer. Just as the World Health Organization proposed in 2003 that malignant tumors should be treated as a chronic disease, the diagnosis and treatment of $\mathrm{ABC}$ have developed rapidly over the past decades. On November 3, 2011, the European College of Oncology (ESO), together with the European Society of Medical Oncology (ESMO), organized global multidisciplinary experts to hold the first International Consensus Conference on ABC in Lisbon, Portugal, and formulated the first edition of the international consensus guidelines for ABC (ABC1) (2). Subsequently, the conference was held every 2 years for guideline updates. The 2011 San Antonio Breast Cancer Symposium (SABCS) further emphasized the concept of chronic disease in the treatment of $\mathrm{ABC}$. The consensus guidelines for $\mathrm{ABC}$ have subsequently been updated every 2 years and are known as $\mathrm{ABC} 2$ (3), $\mathrm{ABC} 3$ (4), ABC4 (5), and ABC5 (6). On November 14, 2019, the fifth International Consensus Conference was held in Lisbon, Portugal. Participants from 94 countries around the world, a total of 1,500 medical personnel and other representatives, attended the meeting. In all, 44 global multidisciplinary experts (including Chinese scholars Professor Xichun $\mathrm{Hu}$ and Professor Binghe $\mathrm{Xu}$ ) discussed and voted on the fifth edition of the International Consensus Guidelines for ABC.

We present the following article in accordance with the Narrative Review reporting checklist (available at http:// dx.doi.org/10.21037/atm-21-1458).

\section{History and evolution of the ABC guidelines}

The international $\mathrm{ABC}$ expert group for this $\mathrm{ABC}$ guideline was established as early as 2005 , due to the reality that, although the survival rate of patients with early breast cancer had been greatly improved over recent years, the survival rate of patients with $\mathrm{MBC}$ had not significantly improved or changed. The ABC1 guideline (2) was intended to establish international evidence-based guidelines for the treatment of $\mathrm{MBC}$ and to highlight areas where clinical research was still needed. The ABC1 guidelines outline the assessment, treatment, supportive care, and palliative care for MBC. The guidelines emphasize the importance of multidisciplinary teams in managing the needs and treatment of MBC patients, including site-specific care and psychological support for metastasis.

The ABC2 guidelines (3) issued in 2014 were updated in various respects, and many new recommendations were provided for the treatment of patients with triple-negative breast cancer (TNBC), HER2-positive breast cancer, and hormone receptor (HR)-positive breast cancer. It included new treatment recommendations for advanced HER2positive breast cancer patients who relapsed shortly after trastuzumab adjuvant therapy. However, more researches exploring the best treatment for special breast cancer, such as those in whom the tumor has spread to the liver, pleural cavity or skin, and the new diagnosed stage IV breast cancer (LABC) were still needed.

As economic status varies across the world, guidelines for application on a global scale may be difficult to produce, nonetheless, the $\mathrm{ABC} 2$ guidelines have near universal relevance, spanning the spectrum of global economic development. This is facilitated by the guidelines making recommendations based on solid evidence and in some cases, providing more economical treatment models. It stressed that counselling and better symptom management were within the reach in many countries, as compared with the out-of-reach costs of expensive drugs.

With the prolonged survival of patients with MBC, treatment-related complications may arise, and patients may 
face greater social and economic hardship.

The ABC3 guidelines (4) suggest that clinicians should focus on humane management; that is, striking a balance between survival and quality of life. Based on breast cancer molecular typing, the $\mathrm{ABC} 3$ described in detail the individual diagnosis and treatment strategies for advanced HER-2 positive, HR-positive, and TNBC. It additionally discussed chemotherapy regimens and Next-Generation Sequencing (NGS).

In addition, considering that some patients with longterm complete remission (CR) may not progression after previous continuous anti-HER2 treatment, in order to avoid increasing the financial burden of patients, the guidelines recommended that treatment should be discontinued if there is a long CR period after treatment, and anti-HER2 therapy should be considered again after disease progressed.

On November 8, 2017, the fourth ESO-ESMO International $\mathrm{ABC}$ Consensus (ABC4) Conference was held in Lisbon, Portugal. In this iteration, it recommended patients should consult the breast specialist and receive professional treatment in a hospital with specialized breast oncology department. Multidisciplinary teams were highlighted in their capacity to provide diagnosis and treatment services. In the whole process of screening, diagnosis, treatment, rehabilitation and follow-up of $\mathrm{MBC}$, the guidelines recommended doctors to make comprehensive plans and carry out whole-process monitoring and management, so as to prolong the survival of patients and improve the quality of life as far as possible, which is also conducive to the mental health of patients. The ABC4 (5) also focused on LABC, indicating that if CR can be achieved through systemic therapy only or combination with local treatment such as surgery or radiotherapy, some MBC patients can survive for a long time and even be cured. The ABC4 guidelines recommended maintenance therapy as the further treatment for these patients.

For first-line treatment in patients with HER2positive $\mathrm{MBC}, \mathrm{ABC} 3$ (4) and $\mathrm{ABC} 4$ (5) have the same recommendation: trastuzumab + pertuzumab + chemotherapy. T-DM1 is recommended as second-line treatment, and lapatinib + capecitabine or lapatinib + trastuzumab targeted therapy is recommended as thirdline treatment. In estrogen receptor (ER)-positive MBC, the $\mathrm{ABC} 4$ generally recommends endocrine therapy (or hormone therapy, HT) as the first-line therapy, while chemotherapy being preferred only for patients with relatively rapid disease progression or visceral crisis. CDK4/6 inhibitors + aromatase inhibitors (AIs) is the most preferred recommendation for first-line treatment. Some patients can be treated with AIs or fulvestrant alone. For second-line treatment, patients who have not previously received CDK4/6 inhibitors may choose CDK4/6 inhibitors plus fulvestrant, or fulvestrant alone, or choose another type of AIs, or ER antagonist, depending on the patient's specific situation. For hereditary TNBC, BRCA1/2 gene testing currently has the most sufficient clinical evidence, and patients with $B R C A 1 / 2$ gene mutations are more sensitive to poly ADP-ribose polymerase (PARP) inhibitors (e.g., olaparib) and platinum-based chemotherapy. Other genes are in the exploratory stage, and their clinical value are unclear. Brain radiation necrosis may occur in some patients following brain radiotherapy, and may aggravate by subsequent treatment. For these patients, ABC4 recommends high-dose steroid and bevacizumab therapy if response is poor. $\mathrm{ABC} 4$ added new information on bisphosphonates for pain relief, the management of nausea and vomiting, the use of granulocyte colony-stimulating factor (G-CSF), and other supportive treatment.

In 2020 the fifth edition of the International Consensus Guidelines for MBC (ABC5) (6) provided updated management recommendations for all breast cancer subtypes, LABC, follow-up, palliative and supportive care, and up-to-date procedures for diagnosis and treatment.

For ER+/HER2-MBC, CDK4/6 inhibitors plus hormone therapy is considered the standard treatment, as the combination regimen significantly improves progressionfree survival (PFS), overall survival (OS), and quality of life (7-9). It is not clear whether CDK4/6 inhibitors should be preferred for first- or second-line treatment. However, most panelists preferred CDK4/6 inhibitors as first-line therapy. It is important to note that there are no head-tohead clinical trials of three different CDK4/6 inhibitors. There is no data to support the use of CDK4/6 inhibitors combined with HT in maintenance therapy. In this setting, HT should be used as maintenance therapy.

For HER2-positive $\mathrm{MBC}$, in third-line treatment and beyond, neratinib + capecitabine versus lapatinib + capecitabine showed a limited PFS benefit, with no significant difference in the OS co-endpoint. In patients with brain metastases, the combination regimen of neratinib has potential efficacy, but more researches are needed (10). Tucatinib + trastuzumab + capecitabine versus trastuzumab + capecitabine in patients previous treated with trastuzumab, pertuzumab, and T-DM1 (including brain metastases) showed a smaller PFS benefit ( 2 months) and OS benefit (4 months), but at the cost of higher toxicity (e.g., 
diarrhea) (11). A phase II study found that DS-8201 showed potential antitumor activity in the multiline treatment of advanced HER $2+$ breast cancer (median treatment line number was 6) (12). Based on these results, DS-8201 has been approved as an effective treatment in this setting. But it's worth mentioning that treatment-related pulmonary toxicity (pulmonary interstitial disease/pneumonia) can be fatal and requires active surveillance and appropriate management.

Atezolizumab + albumin-bound paclitaxel is one of the first-line treatment options for PD-L1 positive mTNBC either newly diagnosed or progression after at least 12 months of (neo)adjuvant chemotherapy $(13,14)$. However, due to the low remission rates, immune checkpoint inhibitors (ICIs) are not routine recommended for post-treatment TNBC.

So far, the $\mathrm{ABC}$ international consensus guideline has been released for almost 10 years in 5 versions. It has a crucial role in raising the level of diagnosis and treatment of patients with $\mathrm{MBC}$ worldwide, so that the survival of $\mathrm{MBC}$ is prolonged gradually. However, the guideline indicates that further high-quality clinical trials on $\mathrm{MBC}$ are still needed.

\section{The hope for cure in MBC}

Being universally known, $\mathrm{MBC}$ is considered as a chronic disease, and a long-period treatment is needed. So, we focus on the balance between curative effect (i.e., disease control) and the quality of life for MBC patients. Once early breast cancer patients occurred recurrence or metastasis, they always concurrently present with systemic metastasis of multiple organs. Therefore, MBC is a systemic spreading disease, and patients should take a comprehensive assessment before treatment to evaluate the prognosis and predict the efficacy. Then, effective and appropriate treatment will be given to make patients achieve partial response (PR), or even CR. However, for those patients with poor tolerance and poor prognosis, compliance and quality of life should be considered priority. Besides, for patients who have gained CR or PR or even stable disease (SD), maintenance treatment given until tumor progression is necessary and important, and targeted drugs, endocrine therapy, or mono-chemotherapy is often taken as maintenance treatment. With this therapeutic concept, some MBC patients with CR are likely to survive a long term, and some gradually transform from systemic disease to local disease. Sometimes, these local lesions can be resolved by local treatment and their prognosis are similar to those who achieve CR. Patients who achieve $\mathrm{CR}$ and survive for a long time are likely to be cured. And this circumstance is not very rare in clinical practice. The international consensus of $\mathrm{ABC}$ experts $(\mathrm{ABC} 1-5)$ has also changed the description of $\mathrm{MBC}$ from incurable to hard to heal. Now, cure is advocated as the objective of $\mathrm{MBC}$ patients, indicating that the cure for $\mathrm{MBC}$ has become possible.

This small number of MBC patients who are most likely to be cured are usually HER-2 positive patients with low tumor burden or HR-positive MBC with non-visceral metastasis. But, for patients with metastatic triple-negative breast cancer (mTNBC), cure remains a big challenge.

At present, there is no standard definition of cure for $\mathrm{MBC}$. Case studies and reviews indicate that cure means MBC patients achieving CR after salvage treatment and maintenance therapy experience a long-term CR status. Their survival patterns are very similar to those early breast cancer patients. The efficacy of MBC was assessed by the 5-year survival rate, and the optimistic overall 5-year survival rate for MBC patients is approximately $30 \%$. If MBC patients with heavy tumor burden, rapid progression disease or some subtype with poor prognosis (e.g., most of mTNBC) were excluded, the 5-year survival rate would be greatly improved. For example, the CLEROPATRA study (15) demonstrates that $37 \%$ of HER-2 positive MBC patients are still alive with first-line dual anti-HER2 targeted treatment after 8 years follow-up (16).

In our opinion, the definition of cure of $\mathrm{MBC}$ is that MBC patients achieve CR and remain for more than five years after systemic treatment, including those with local therapy (e.g., surgery or radiotherapy, et al.). Therefore, it is very important for MBC patients to make the tumor rapidly shrink through effective treatment, especially to achieve CR.

\section{Our explorations and achievements in the MBC}

Since 2015, Chinese multidisciplinary experts have drafted Chinese guidelines for clinical diagnosis and treatment of $\mathrm{ABC}$. Till now, we have 4 editions which have been also recognized by international experts. These guidelines further improved the survival and the quality life of $\mathrm{MBC}$ patients both in China and all over the world (17-19). At the Department of Breast Oncology in Zhejiang Cancer hospital, about $80 \%$ of the breast cancer patients are MBC patients. And since 2000, classification therapy guided by molecular classification has become the standard 
strategy Because of the high heterogeneity of breast cancer. Consequently, we have conducted a series of researches and meaningful explorations in different subtypes of MBC.

\section{HER-2 positive MBC}

HER-2 positive type is a special subtype, accounting for approximately $25 \%$ of breast cancer. In recent years, survival have been significantly improved due to the widespread application of anti-HER-2 targeted therapy. The PFS and OS of first-line chemotherapy combined with trastuzumab are 12 months and 30 to 40 months, respectively. And amazingly, PFS and OS are prolonged to 18.7 and 57.1 months, respectively, when dual anti-HER-2 targeted regimen consisting of trastuzumab and pertuzumab is given in the first-line treatment. However, primary or secondary resistance to anti-HER2 treatment still exists, with the mechanism underlying this resistance being rather complex. The HER2 extracellular domain (ECD) is the extracellular domain of the HER-2 protein on the surface of breast cancer cells. It may be cleaved and shed from the surface of breast cancer cells, and tumor cells may become resistant to trastuzumab, and serum ECD can be detected by enzyme-linked immunosorbent assay (ELISA).

In the 2012 SABCS, we reported a prospective study in which $207 \mathrm{MBC}$ patients were enrolled at Zhejiang Cancer Hospital from March 2009 to July 2011(20). We explored he relationship between circulating ECD and clinical pathological characteristics. Serum HER2 ECD was measured by ELISA assay. Tissue HER2 status was determined by immunohistochemistry (IHC) and fluorescence in situ hybridization (FISH) test in HER2++ cases. ECD levels were evaluated to predict the outcome and their values as prognostic markers. For MBC patients, the percentage of high level serum ECD $(\geq 15 \mathrm{ng} / \mathrm{mL})$ was $39.1 \%$ (43/110) in HER2-positive cases and 23.4\% (22/94) in HER2-negative cases ( $\mathrm{P}=0.017)$; high ECD levels $(\geq 15 \mathrm{ng} / \mathrm{mL})$ were significantly associated with elevated serum carcino-embryonic antigen (CEA) $(52.1 \%$ vs. $21.5 \%$, $\mathrm{P}=0.000)$, carcinoantigen 153 (CA153) (53.2\% vs. $17.1 \%$, $\mathrm{P}=0.000$ ), and $\mathrm{CA} 125$ (48.5\% vs. $23.5 \%, \mathrm{P}=0.000$ ). This correlation was also seen in lactic acid dehydrogenase (LDH) (53.3\% vs. $23.1 \%, \mathrm{P}=0.000)$ and alkaline phosphatase (AKP) $(51.2 \%$ vs. $26.5 \%, \mathrm{P}=0.002)$. Besides, increased ECD was also related to vital visceral metastasis $(37.9 \%$ vs. $14.8 \%, \mathrm{P}=0.002$ ), liver ( $42.7 \%$ vs. $24.0 \%, \mathrm{P}=0.005$ ), brain (50.0\% vs. $26.7 \%, \mathrm{P}=0.003)$. This study reveals that regular circulating ECD assay in MBC patients can provide the real-time monitoring of tumor burden and prediction of poor outcome, and may offer an important opportunity to reassess HER2 status in HER2-negative MBC patients.

\section{HR-positive MBC}

HR positive (HR+) HER2 negative (HER2-) are the most common types of $\mathrm{MBC}$, accounting for approximately twothirds of breast cancer. Most HR+ HER2- patients are endocrine therapy-sensitive, and often have long diseasefree survival (DFS), with no visceral crisis, remaining hormone-positive status in metastatic lesions. Therefore, endocrine therapy should be preferred as a first-line treatment in most patients. While there are still a small portion of patients who are relatively insensitive to endocrine therapy or have visceral crisis or have high tumor burden need combined chemotherapy as their first choice. Maintenance therapy strategies followed by combined chemotherapy include chemotherapy and endocrine therapy, but there is no clinical evidence supporting either as the optimal choice. Chen et al. (21) conducted a retrospective single-center analysis of $138 \mathrm{MBC}$ patients with stable disease after capecitabine-based chemotherapy recruited from 2003 to 2012. And the data showed that HT was superior to capecitabine alone as maintenance treatment (PFS 13.0 vs. 8.0 months, $\mathrm{P}<0.011$ ). Li et al. (22) also completed a retrospective study in which MBC patients received docetaxel combined with capecitabine as the first-line treatment from 2008 to 2013, and the endocrine maintenance therapy showed longer PFS than capecitabine maintenance (11.5 vs. 6.1 months, respectively).

In the 2020, SABCS, we reported the primary analysis results of the OVERSTEP (NCT02597868) trial (23), a multicenter, randomized clinical trial evaluating the effectiveness and security of capecitabine or endocrine therapy as a maintenance therapy regimen after first-line chemotherapy with capecitabine combined regimen in HRpositive and HER2-negative MBC patients. The first patient was enrolled on June 5, 2013 and the last one was enrolled on January 9, 2019. These patients received capecitabine plus other chemotherapy as first-line salvage chemotherapy for at least 4 cycles. The patients with CR, PR, or stable disease $(\mathrm{SD})$ were randomized in a 1:1 ratio to receive either capecitabine alone or HT as maintenance therapy. The primary endpoint was PFS after randomization. In the report, we showed that the median PFS was 17.5 months in the HT maintenance group which was significantly longer than that in the capecitabine maintenance group 
with 12.2 months $(\mathrm{P}=0.013)$. Besides, in the endocrinesensitive group, the median PFS for the HT maintenance group was obviously longer when compared with that for the capecitabine maintenance group (29.3 vs. 14.8 months, $\mathrm{P}=0.042$ ). In addition, in the non-visceral metastasis group, the median PFS for the HT maintenance group was longer than that for the capecitabine maintenance group (25.3 vs. 17.0 months, $\mathrm{P}=0.037)$. However, in the endocrine-resistant group, there was no difference of PFS between two cohorts.

These findings suggest that HT maintenance is the priority choice for HR+/HER2 - MBC after first-line combined chemotherapy, especially for HT-sensitive and non-visceral involved cases.

On July 26, 2015, fulvestrant (500 mg) was approved in China, and entered the national health insurance in July 2017. In recent years, fulvestrant has come to be widely applied in HR-positive MBC.

We conducted a retrospective study of HR+/HER2MBC patients who received $500 \mathrm{mg}$ of fulvestrant (24). Overally, 147 patients were recruited between September 2014 and June 2017. All participants received a fulvestrant loading-dose regimen $[500 \mathrm{mg}$, intramuscular (IM), every 28 days], and premenopausal patients also received goserelin or leuprorelin. With a median follow up of 20 months, the median PFS was 7.2 months (95\% CI: 6.0-8.3 months), and the OS was immature; $30.6 \%(45 / 147)$ were at first line and $69.4 \%(102 / 147)$ were at second line or later. PFS of the first line was significantly longer than that of the second line or later (15.9 vs. 6.1 months; $\mathrm{P}=0.000$ ). In premenopausal women, the PFS was 9.5 months, which was similar to that in postmenopausal women $(7.0$ months $)(\mathrm{P}=0.757)$. The ORR and CBR were $8.2 \%$ and $81.8 \%$, respectively, with no CR and 28 (19.0\%) PD cases. The most common adverse events were injection site pain $(8.0 \%)$ and musculoskeletal disorders $(7.0 \%)$. This real-word study demonstrated that fulvestrant was highly effective and well tolerated in the patients with $\mathrm{HR}+/ \mathrm{HER} 2-\mathrm{MBC}$. Additionally, fulvestrant combined with ovarian suppression was also an active option for premenopausal HR+/HER2- MBC.

BOLERO-2 trial showed a significant advantage in terms of PFS and response rate (RR) of everolimus and exemestane in HER2-negative MBC progressing after a non-steroidal aromatase inhibitor treatment. However, subsequent retrospective studies showed the poor tolerability of the high dose everolimus $(10 \mathrm{mg} / \mathrm{d})$ and a high rate of dose reduction or interruption. Thus, we evaluated the efficacy and safety of low-dose everolimus $(5 \mathrm{mg} / \mathrm{d})$ combined with hormone therapy (HT) in Chinese HR- positive, HER2-negative MBC patients, which is reported in the 2018 SABCS (15). In all, $68 \mathrm{HR}+/ \mathrm{HER} 2-\mathrm{MBC}$ patients with low dose everolimus $(5 \mathrm{mg} / \mathrm{d})$ and $\mathrm{HT}$ who were admitted to Zhejiang Cancer Hospital between September 2014 and September 2017 were included in this exploratory study. The results indicated that 16 (23.5\%) were at the first or second line, and $52(76.5 \%)$ were at third line or later. After a median follow-up of 14 months, PFS was 5.3 months, and the OS was immature. PFS for the front lines (the first and second line) was significantly longer than that of the third line and later (12.9 vs. 4.6 months; $\mathrm{P}=0.009)$. No patient reached CR, $11(16.2 \%)$ achieved PR, 42 (61.7\%) had SD, and 15 (22.1\%) had PD. The ORR and CBR were $16.2 \%$ and $78.9 \%$, respectively. The most common adverse events (all grade) were stomatitis (26.5\%), fatigue $(10.0 \%)$, infection $(11.8 \%)$. This exploratory study revealed that everolimus (low-dose) combined with hormone therapy was effective for HR-positive and HER2negative MBC patients, especially in the front lines. The adverse event profiles were similar to previous studies, but the incidences were decreased.

\section{$m T N B C$}

TNBC has the worst prognosis and poorest treatment efficacy among all breast cancer subtypes and accounts for about $15-20 \%$ of all breast cancers. The median distant disease-free interval for relapsed TNBC is about 1-2 years. For the mTNBC, the PFS of first-line treatment is approximately $5-8$ months, and the median OS is about 1 year. For quite some time, no targeted drugs were available for these patients, only the conventional cytotoxic antitumor drugs like anthracycline, taxane, capecitabine, gemcitabine, vinorelbine, platinum, etc. With the gradual clarification of the molecular characteristics of mTNBC in recent years, antiangiogenic agents, PARPi, nab-paclitaxel, PI3K/AKT/ mTOR pathway inhibitors, androgen receptor antagonists, and immunotherapy have been explored and applied. Previously, our team conducted several small meaningful clinical studies of antiangiogenic agents in mTNBC.

Thalidomide (THA) has antitumor effects such as angiogenesis, improvement of immune function, and cachexia of patients with advanced tumors. The efficacy and safety of THA in patients with MBC are still being investigated. At the most recent Global Academic Programs (GAP) meeting, we reported a preliminary analysis of a phase 2 study (25). There are 9 patients received median 10 cycles (2-24 cycles) of THA. No patients experienced 
a dose reduction of THA. The median follow-up time was 10 months, and the median PFS was 7.0 months $(95 \% \mathrm{CI}$ : 3.9-9.7 months). The best response reached was CR in 1 patient, while PR occurred in 5 patients. Adverse reactions were mainly dry mouth $(11.1 \%$, grade 1$)$ and constipation (66.7\%, grade 1); 1 patient experienced a grade 3 pulmonary embolism needing hospitalization, with this severe adverse event (SAE) possibly being THA related. Three patients developed disease progression and no deaths occurred during follow-up. Thus, THA exhibited potential efficacy and was well tolerated in pretreated patients, but clinicians should be wary of thrombus events in practice.

Rh-endostatin (endostar), a recombinant human endostatin, is a potent inhibitor of angiogenesis. Endostar combined with chemotherapy was found to be effective and well tolerated for the treatment of HER2-negative MBC, especially for TNBC. Its efficacy and safety could be further studied in randomized trials. The Prometheus study (26) was a single-arm multicenter phase 2 trial designed to assess the efficacy and safety of endostar combined with chemotherapy in HER2-negative MBC. A total of 40 female patients were enrolled in 3 centers. Ten patients were previously untreated, thirty patients had at least 1 line of chemotherapy for advanced disease. For subtype, 31 patients were TNBC and 9 patients were HR positive. Platinum combined with paclitaxel or gemcitabine was administered with endostar in $55 \%$ of patients. Others received paclitaxel or gemcitabine combined with endostar. Among 30 patients with measurable disease, the ORR was $40 \%$ and the disease control rate (DCR) was $83 \%$. The ORR for patients treated the first-line therapy was $75 \%$, while that for second line or beyond was $27 \%$. For triplenegative and HR-positive breast cancer patients, the ORR was $46 \%$ and $17 \%$, respectively. There is an urgent need to improve the efficacy of chemotherapy for HER2-negative patients who lack anti-HER2 therapies. These studies reviewed here are an exploration of the treatment of triplenegative MBC or HER2-negative MBC with antiangiogenic drugs.

\section{Discussion}

Overall, the treatment of $\mathrm{MBC}$ is exceedingly challenging, with many subtypes, complex conditions in the treatment of patients, and a wide variety of antitumor drugs. Survival is strongly related to subtypes, with the major advances seen in HER2-positive MBC. Arguably, the CLEOPATRA study has set the standard of first-line treatment of HER2- positive MBC, with the outlook for achieving a cure in HER2-positive MBC being optimistic. In the past decade, our conception of $\mathrm{MBC}$ has moved from incurable, to difficult to cure, to the belief that some patients can indeed be cured. This reflects the achievements of core conceptual essential shifts in the treatment of MBC.

The MBC patients who have reached CR with effective treatment usually get a long-term survival. And some patients maintain CR status for more than 5 years, which can be concluded that this subtype of MBC patients have reached clinical cure. For instance, in clinical practice once HER2-positive MBC patients' CR status persists for 3 to 5 years, they are allowed to discontinue targeted treatment.

However, even if the above arguments are common sense, the clinical cure standard or definition for $\mathrm{MBC}$ still lacks credible clinical data support. Our concepts and practices will contribute to the design of relevant clinical research and accumulation of evidence, and consequently, more MBC patients can hope for a cure.

\section{Acknowledgments}

Funding: This work was supported by grants from the Key Project of Science and Technology Program of Zhejiang Province (No. 2012C13019-1), Natural Science Foundation (No.81672597), Zhejiang Provincial Natural Science Foundation (No. Y2101312 and LY17H160041), the Zhejiang Provincial Medical Science and Technology Program (No. 2021KY551).

\section{Footnote}

Reporting Checklist: The authors have completed the Narrative Review reporting checklist. Available at http:// dx.doi.org/10.21037/atm-21-1458

Conflicts of Interest: All authors have completed the ICMJE uniform disclosure form (available at http://dx.doi. org/10.21037/atm-21-1458). The authors have no conflicts of interest to declare.

Ethical Statement: The authors are accountable for all aspects of the work in ensuring that questions related to the accuracy or integrity of any part of the work are appropriately investigated and resolved.

Open Access Statement: This is an Open Access article distributed in accordance with the Creative Commons 
Attribution-NonCommercial-NoDerivs 4.0 International License (CC BY-NC-ND 4.0), which permits the noncommercial replication and distribution of the article with the strict proviso that no changes or edits are made and the original work is properly cited (including links to both the formal publication through the relevant DOI and the license). See: https://creativecommons.org/licenses/by-nc-nd/4.0/.

\section{References}

1. Deluche E, Antoine A, Bachelot T, et al. Contemporary outcomes of metastatic breast cancer among 22,000 women from the multicentre ESME cohort 2008-2016. Eur J Cancer 2020;129:60-70.

2. Cardoso F, Costa A, Norton L, et al. 1st International consensus guidelines for advanced breast cancer (ABC 1). Breast 2012;21:242-52.

3. Cardoso F, Costa A, Norton L, et al. ESO-ESMO 2nd international consensus guidelines for advanced breast cancer (ABC2)dagger. Ann Oncol 2014;25:1871-88.

4. Cardoso F, Costa A, Senkus E, et al. 3rd ESO-ESMO International Consensus Guidelines for Advanced Breast Cancer (ABC 3). Ann Oncol 2017;28:3111.

5. Cardoso F, Senkus E, Costa A, et al. 4th ESO-ESMO International Consensus Guidelines for Advanced Breast Cancer (ABC 4)dagger. Ann Oncol 2018;29:1634-57.

6. Cardoso F, Paluch-Shimon S, Senkus E, et al. 5th ESOESMO international consensus guidelines for advanced breast cancer (ABC 5). Ann Oncol 2020;31:1623-49.

7. Slamon DJ, Neven P, Chia S, et al. Overall Survival with Ribociclib plus Fulvestrant in Advanced Breast Cancer. N Engl J Med 2020;382:514-24.

8. Finn RS, Martin M, Rugo HS, et al. Palbociclib and Letrozole in Advanced Breast Cancer. N Engl J Med 2016;375:1925-36.

9. Sledge GW, Jr., Toi M, Neven P, et al. MONARCH 2: Abemaciclib in Combination With Fulvestrant in Women With HR+/HER2- Advanced Breast Cancer Who Had Progressed While Receiving Endocrine Therapy. J Clin Oncol 2017;35:2875-84.

10. Saura C, Oliveira M, Feng YH, et al. Neratinib Plus Capecitabine Versus Lapatinib Plus Capecitabine in HER2-Positive Metastatic Breast Cancer Previously Treated With $\geq 2$ HER2-Directed Regimens: Phase III NALA Trial. J Clin Oncol 2020;38:3138-49.

11. Murthy RK, Loi S, Okines A, et al. Tucatinib, Trastuzumab, and Capecitabine for HER2-Positive Metastatic Breast Cancer. N Engl J Med 2020;382:597-609.
12. Modi S, Saura C, Yamashita T, et al. Trastuzumab Deruxtecan in Previously Treated HER2-Positive Breast Cancer. N Engl J Med 2020;382:610-21.

13. Schmid P, Rugo HS, Adams S, et al. Atezolizumab plus nab-paclitaxel as first-line treatment for unresectable, locally advanced or metastatic triple-negative breast cancer (IMpassion130): updated efficacy results from a randomised, double-blind, placebo-controlled, phase 3 trial. Lancet Oncol 2020;21:44-59.

14. Schmid P, Adams S, Rugo HS, et al. Atezolizumab and Nab-Paclitaxel in Advanced Triple-Negative Breast Cancer. N Engl J Med 2018;379:2108-21.

15. Shao X, Zheng Y, Chen ZH, et al. Efficacy and safety of low-dose everolimus in Chinese HR-positive, HER2negative metastatic breast cancer patients. Journal of Clinical Oncology 2018;36:e13035-e.

16. Swain SM, Miles D, Kim SB, et al. Pertuzumab, trastuzumab, and docetaxel for HER2-positive metastatic breast cancer (CLEOPATRA): end-of-study results from a double-blind, randomised, placebo-controlled, phase 3 study. The Lancet Oncology 2020;21:519-30.

17. Chinese Anti-Cancer Association, Committee of Breast Cancer Society. Chinese expert consensus on the clinical diagnosis and treatment of advanced breast carcinoma(2018). Zhonghua Zhong Liu Za Zhi 2018;40:703-13.

18. Breast Cancer Expert Committee of National Cancer Quality Control Center; Breast Cancer Expert Committee of China Anti-Cancer Association; Cancer Drug Clinical Research Committee of China Anti-Cancer Association. Guidelines for clinical diagnosis and treatment of advanced breast cancer in China (2020 Edition). Zhonghua Zhong Liu Za Zhi 2020;42:781-97.

19. $\mathrm{Xu} \mathrm{B}, \mathrm{Hu} \mathrm{X}$, Feng J, et al. Chinese expert consensus on the clinical diagnosis and treatment of advanced breast cancer (2018). Cancer 2020;126 Suppl 16:3867-82.

20. Wang X, Shao XY, Xu XH, et al. Abstract P2-05-05: Circulating HER2 extracellular domain (ECD) predicts a poor prognosis for metastatic breast cancer patients. Cancer Res 2012;72 Suppl 24.

21. Chen XL, Du F, Hong RX, et al. Hormonal therapy might be a better choice as maintenance treatment than capecitabine after response to first-line capecitabine-based combination chemotherapy for patients with hormone receptor-positive and HER2-negative, metastatic breast cancer. Chin J Cancer 2016;35:39.

22. Liang X, Yan Y, Wang L, et al. First-line chemotherapy with docetaxel plus capecitabine followed by capecitabine 
or hormone maintenance therapy for the treatment of metastatic breast cancer patients. Oncol Lett 2015;9:987-93.

23. Huang J, Shao X, Cai L, et al. Abstract PS13-01: Primary analysis of OVERSTEP: A multicenter, randomized clinical trial of capecitabine or endocrine therapy as a maintenance therapy after the 1st-line chemotherapy in hormone receptor positive and HER2-negative advanced/metastatic breast cancer. Cancer Res 2021;81:PS13-01.

24. Shao X, Jia WX, Zheng Y, et al. Efficacy and safety of fulvestrant $500 \mathrm{mg}$ in Chinese HR-positive, HER2-

negative metastatic breast cancer patients: A real-world

Cite this article as: Wang X, Shao X, Huang J, Lei L, Huang Y, Zheng Y, Cao W, Chen Z. Exploring the concepts and practices of advanced breast cancer treatment: a narrative review. Ann Transl Med 2021;9(8):721. doi: 10.21037/atm-21-1458 study. J Clin Oncol 2018;36:e13046.

25. Lei L. Thalidomide Plus Chemotherapy: A Phase II Study in Advanced Breast Cancer- Interim analysis. GAP2018, abstr 4069.

26. Huang Y, Zheng Y, Cao WM, et al. A phase II study of rh-endostatin in combination with chemotherapy in human epidermal growth factor receptor 2 (HER2) negative advanced breast cancer (ABC). J Clin Oncol 2020;38:abstr 1071 .

(English Language Editor: J. Gray) 